\title{
A Simple and Effective Heuristic Control System for the Heliostat Field of Solar Power Tower Plants
}

\section{Nicolás C. Cruz ${ }^{1}$, José Domingo Álvarez ${ }^{1}$, Juana L. Redondo ${ }^{1}$, Manuel Berenguel $^{1}$, Ryszard Klempous ${ }^{2}$, Pilar M. Ortigosa ${ }^{1}$}

${ }^{1}$ University of Almería, ceiA3 Excellence Agrifood Campus, Department of Informatics, Sacramento Road, n/a, 04120, La Cañada de San Urbano, Almería, Spain,ncalvocruz@ual.es, jhervas@ual.es, jlredondo@ual.es, beren@ual.es, ortigosa@ual.es

${ }^{2}$ Wrocław University of Science and Technology, Department of Electronics, wybrzeże Stanisława Wyspiańskiego 27, 50-370 Wrocław, Polonia, ryszard.klempous@pwr.edu.pl

\footnotetext{
Abstract: Solar power tower plants use large arrays of mirrors, known as heliostats, to concentrate solar radiation on their receiver and heat the working fluid inside them. However, receivers must not be under thermal stress. Otherwise, their life expectancy is reduced, which affects the cost and viability of production plant. Controlling the flux distributions on receivers requires selecting the active heliostats and their target points. It is a challenging task that should not be under the responsibility and expertise of human operators only. This work defines a closed-loop controller to keep the setpoint or desired flux map under certain conditions. It combines real measurements and an ad-hoc analytical model of the target field with a set of heuristic rules that covers how to activate, deactivate, and re-aim heliostats. The proposed system has been applied to a model of the CESA-I field at the Solar Platform of Almería. The open-source ray-tracer Tonatiuh represents the reality. The initial operation point has been determined with a theoretical flux distribution optimizer. According to the experimentation, the controller improves the initial and modelbased flux distribution by raising its power from 708.4 to $736.4 \mathrm{~kW}$ (with a setpoint of $739.6 \mathrm{~kW}$ ).
}

Keywords: solar power tower plant; heliostat field; flux distribution; automatic control; closed-loop control; heuristics

\section{Introduction}

The pollution and depletion problems associated with electricity generation through traditional fuel-based technologies have increased the interest in renewable energy [1-3]. Concentrated Solar Power systems (CSP) are especially 
attractive because of their hybridization capabilities, as well as, their production stability through thermal storage [4]. Among the different CSP technologies [1, 5], Solar Power Tower plants (SPT) are probably the most promising ones because the high temperatures reached result in high thermodynamic efficiency $[2,4]$ and less thermal storage requirements [2]. SPT plants have good development prospects linked to improving their commercial competitiveness $[2,6]$ and also attracts the interest of this work.

Conceptually, a SPT plant consists of a solar radiation receiver linked to a power block and a set of solar tracking mirrors known as heliostats. The heliostats follow the apparent movement of the Sun to concentrate the incident solar radiation on the receiver. The receiver, which is generally (but not necessarily [7]) on top of a tower for better focusing, contains a circuit for a Heat-Transfer Fluid (HTF). The goal is to heat the HTF with the power that the field concentrates on the receiver. Once the temperature of the HTF is appropriate, it serves to generate electricity in a power cycle (either combined, gas or steam turbine cycle). The HTF can also be stored for delivery under demand. For instance, the Gemasolar plant can generate electricity approximately for approximately 15 hours without solar radiation [2]. Figure 1 shows a simplified representation of a SPT plant with a steam turbine cycle. There exist different variations over the basis described starting from the receiver design (e.g., flat, cylindrical) and the HTF (e.g., molten salt, air, water) [4]. The interested reader can find more information about SPT plants in [8,9].

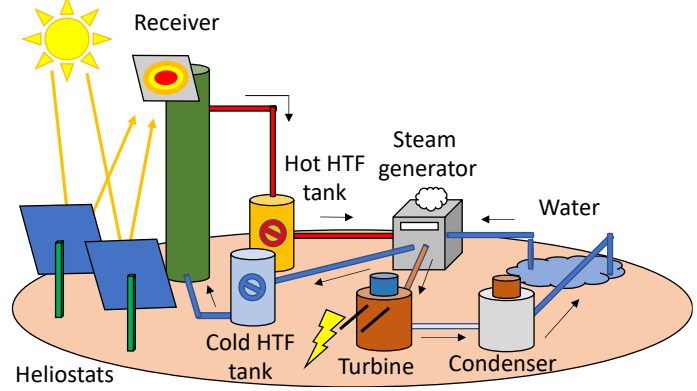

Figure 1

Simple depiction of a solar power tower plant with a steam turbine cycle

Despite its conceptual simplicity, the radiation receiver of a SPT plant is a sophisticated, expensive and fragile component, that needs special care to reduce the production costs [10] and maximize throughput [11]. The solar flux distribution that the heliostat field reflects on its receiver may cause thermal stress, premature aging, and deformation [10-12]. It is also directly related to the production efficiency of the plant as the interception factor, i.e., the ratio of nonprofited radiation $[10,13,14]$. Therefore, it is necessary to control the flux distribution reflected by the field to keep the receiver safe while concentrating as much power as possible $[15,16]$. For this reason, the development of optimal aiming strategies attracts the attention of many researchers [16]. 
The flux distribution achieved on the receiver depends on the active heliostats and their aim points [17-19]. Besides, it is affected by the apparent solar movement, the evolution of direct normal irradiance (DNI) throughout the day (including its sudden variations caused by clouds [15]), heliostat errors, the wind and other atmospheric phenomena $[14,15,17]$. Consequently, there are far too many variables to delegate the aiming control tasks to human operators [17], especially considering the development of current multi-aiming strategies [18, 20]. Many proposals have been made to control heliostat fields with different objectives and scopes [21].

This work presents a feedback control system connected to a flux optimization method. The optimizer theoretically configures the field to obtain any desired flux distribution. The controller minimizes the error between the flux distribution achieved after translating the theoretical result to reality and the desired one. The control logic is based on heuristic rules. It tries to reduce the effect of disturbances, modelling and optimization errors. The results presented in this paper show how the feedback controller improves the initial flux distribution computed with model-based optimization.

The paper is structured as follows: Section 2 contains a literature review of recent aiming methods. Section 3 summarizes the technical background of the control logic described in this work. Section 4 describes the heuristic closed-loop controller designed. Section 5 explains the experimentation carried out. Finally, the last section contains the conclusions and future work lines.

\section{Literature Review}

Salomé et al. [10] implement a TABU local search algorithm [22] to aim heliostats by following a combinatorial formulation of heliostats with a finite set of aim points. They design an open-loop controller that tries to obtain homogeneous flux distributions with acceptable spillage. Distant heliostats are forced to aim at central zones to reduce spillage. Besarati et al. [13] design a genetic algorithm [23] with a similar combinatorial approach to achieve flat flux forms by minimizing their standard deviation. The authors also add a dedicated component to reduce extreme spillage situations. Grobler [14] combines the two previous strategies by using the TABU search for generating initial solutions for the genetic optimizer. The overall goal is the same, but the descriptiveness of the objective function of the optimization problem is improved. Yu et al. [24] use a TABU search to minimize flux peaks and spillage by following a combinatorial formulation. Heliostats are grouped to reduce the search space, but the shape of the receiver is considered in depth to distribute the aim points. 
Belhomme et al. [25] apply ant colony optimization [26] to assign the best aim point to each heliostat in a combinatorial context. The method avoids dangerous radiation peaks, but its focus is on maximizing the performance of the receiver. Maldonado et al. [27] compare the previous method to a new proposal of local scope that studies small variations to solutions. The ant colony optimizer is more robust in general, while the local one, can achieve high-quality solutions. The authors finally suggest a hybrid method that uses the ant colony to get promising initial solutions for the local method.

Ashley et al. [15] maximize the power on the receiver while keeping it in a valid range and looking for uniformity. The approach is combinatorial, and their integer programming method can find valid solutions in almost real-time, which would allow handling the effect of clouds. This aspect is covered in [28].

Astolfi et al. [11] focus on avoiding flux peaks, and the field is divided into circular sectors to adjust the aim point height of each zone. The continuous optimization problem faced focuses on the vertical aim point and grouping results. The authors test several variations considering overlapping and not doing so, which is less effective. Sánchez-González et al. [16] also consider dividing the field into sectors to achieve flat flux distributions by adjusting aim point heights. The approach relies on exploiting the symmetry of the desired flux distribution and balancing the up and bottom zones. The formulation is based on the concept of aiming factor, which allows estimating the size of the beam reflected by each heliostat. That concept was previously introduced by the authors in [20], where they design a method of two stages to maximize the thermal power output of the receiver while preserving its integrity.

Kribus [29] focuses on avoiding tracking and aiming errors, which is not addressed with open-loop approaches [10] and reduces the dependency on models. The system uses CCD cameras to detect the heliostats not correctly aimed and to calculate the correction signal. Convery [30] also opts for a closed-loop controller, but the design is especially innovative and cheap. It uses piezoelectric oscillators and photodiodes instead of cameras to identify misaiming heliostats. The oscillators serve to make each heliostat vibrate at a unique frequency detected by the photodiodes. Freeman et al. [31] try to improve the standalone capabilities of their closed-loop system. They aim to reduce the necessity of feedback from the receiver of the two previous methods. To this end, they add accelerometers and gyroscopes to the context proposed in [30]. Obtaining specific flux profiles is not covered.

Gallego et al. [32] try to obtain flat flux distributions while maximizing the incident power on the receiver. Instead of defining a combinatorial problem, as usual, the authors opt for a continuous one with two variables per heliostat. The problem is divided into simpler instances by working with different groups of heliostats, called agents, to overcome the computational expenses. The method iteratively considers the effect of groups on each other. 
Finally, in [17], the authors of this paper design a general method that works in a continuous search space like the previous one, but it aims to replicate any desired flux distribution on the receiver. Therefore, it allows avoiding peaks or achieving any other feature by adjusting the flux map reference. It has two layers. The first layer serves to select the active heliostats through a genetic algorithm. The second layer adjusts their aim points by using a gradient descent method. This paper combines that basis with the modelling approach described in [33] to create an operational framework. In this context, a closed-loop controller based on heuristic rules is designed and tested. It aims to make it possible to translate and polish the instantaneous result of the method in [17]. The open-source ray-tracer Tonatiuh $[34]$ is taken as the reality.

\section{Overview of the Operational Framework}

Since the designed method extends the technical context proposed in [17, 33], this section summarizes both for the sake of completeness. Section 3.1 explains how the target field is modelled, and Section 3.2 describes the optimizer that computes off-line field configurations. The interested reader is referred to the complete works for further information.

\subsection{On Predicting the Behavior of the Target Field}

Aim point optimization requires predicting the flux profile over the receiver surface, i.e., optical modelling [3, 10, 14]. It serves to guide the search by estimating the performance of different solutions (linked to off-line optimization [17]) and to predict the effect of different actions (related to on-line control tasks).

As summarized in [13], it is possible to compute flux maps either numerically or analytically. The numerical approach consists in simulating multiple rays through several optical stages to study their interaction with the environment. It is known as ray-tracing and can be done with software packages such as STRAL, SolTrace and Tonatiuh [3, 13, 21]. The analytical methods model the flux maps with mathematical functions such as circular Gaussian distributions as HFLCAL [13, 21].

Ray-tracing offers higher accuracy and flexibility than analytical methods at the expense of higher computational requirements. Considering potential time constraints and the fact that analytical errors attenuate according to the central limit theorem [14], the analytical approach is generally preferred $[11,13,16]$. The work in [25] is an exception example because the authors use ray-tracing and reduce its time impact by storing partial results. Regardless, creating databases with pre-computed information is also an option with analytical strategies $[10,11$, 14, 24]. 
This work relies on an analytical model of the target field. It has been developed by following the methodology proposed by the authors of this paper in [33]. The method defines how to build an ad-hoc model based on accurate data either from ray-tracing or reality. It consists of the following steps:

1. It is necessary to register the position of a subset of heliostats covering all the zones of the target field. Next, the paths of the sun at the location of the field are sampled in a similar way. Finally, the flux map of each heliostat is gathered for each solar position. The maps can come from real measurement or ray-tracing. Notice that since the model under construction will be based on this information, its acquisition conditions will bound the prediction capabilities. For this reason, as long as the flux maps exhibit atmospheric attenuation and shading and blocking losses, the resulting model will implicitly try to consider them.

2. An analytical expression that can describe the flux map that any heliostat projects on its receiver must be selected. As said, Gaussian functions are popular for this purpose. The one chosen is explained later. After that, each of the flux maps previously gathered must be fitted to the expression. The parameters that define the overall shape must be recorded and linked to the particular heliostat and solar position.

3. The set of records is randomly split into a modelling set and a validation one. After that, the goal is to build a model that can predict the aforementioned shape parameters depending on the coordinates of the heliostats and the solar position. Any modelling technique, such as Neural Networks [35] or Random Forests [36], which is the choice in [33], can be used. The validation subset is ignored at this step.

4. It is necessary to confirm the effectiveness of the model or parameter predictor built at the previous step. To this end, it is used to estimate the shape parameters of the records left for validation. The predictions should be similar to the values gathered during the second step, from the original flux maps.

5. Finally, the model can be applied for predicting the behavior of the field, which includes the effect of control actions, as in this case. Concisely speaking, the field model consists of an expression to represent flux maps and an overlying model (known as 'meta-model' in [33]) to adjust its shape parameters on demand.

The target field for which the proposed controller has been designed is the one used to test the methodology introduced in [33], and it is described in Section 5. The analytical expression used to describe the flux map that a certain heliostat $h$ projects on the receiver, $f_{h}$, is the bi-variant Gaussian distribution shown in Equation 1. It has been selected because of its flexibility to directly model noncircular maps, which is coherent with the observations made in $[14,32]$. 
$f_{h}=\frac{P}{2 \pi \sigma_{x} \sigma_{y} \sqrt{1-\rho^{2}}} e^{\left(-\frac{1}{2\left(1-\rho^{2}\right)}\left(\frac{\left(x-\mu_{x}\right)^{2}}{\sigma_{x}^{2}}+\frac{\left(y-\mu_{y}\right)^{2}}{\sigma_{y}^{2}}-\frac{2 \rho\left(x-\mu_{x}\right)\left(y-\mu_{y}\right)}{\sigma_{x} \sigma_{y}}\right)\right)}$

$P$ is the estimated total power contribution of the heliostat. It is measured in $\mathrm{kW}$ and can be scaled to reflect changes in DNI and unpredictable situations, such as, soiling. The variable $\rho$ is the correlation between dimensions $\mathrm{X}$ and $\mathrm{Y}$ of the receiver. The variables $\sigma_{x}$ and $\sigma_{y}$ correspond to the standard deviation along $\mathrm{X}$ and $\mathrm{Y}$, respectively. These four parameters define the overall flux shape on the receiver, and the model can compute them for every heliostat of the field at any solar position. This information is generated depending on the positions of the Sun and the heliostat and stored in a preliminary step. Regarding $\mu_{x}$ and $\mu_{y}$, which are the means from a mathematical point of view, they are linked to the coordinates of the aim point of the heliostat. They are under the control of the method outlined in Section 3.2 first and under that of the controller described in Section 4 at the end.

Although the analytical expression which models flux distributions (Equation (1)) is the same as the one used in [17], the procedure described herein for estimating its parameters also considers the solar position, i.e., time. This aspect was not required for the instantaneous scope of that method, but it is mandatory for designing a complete controller.

\subsection{On Accurately Configuring the Heliostat Field}

The method designed in [17] by the authors of this paper for replicating any flux distribution on the receiver, at a given time (solar position), will be known as the model-based flux optimizer. It benefits from the analytical formulation of the flux maps projected by the heliostats of the field to compute off-line a configuration that replicates any given reference. This approach aims to be general because it permits looking for any flux feature (e.g., homogeneity or low spillage) by defining the appropriate reference.

Its design is based on solving a large-scale and continuous optimization problem. The objective function to minimize measures the difference between the reference and the result achieved according to the field optical model. The resolution method combines two separate layers to select and aim the heliostats.

The first layer is responsible for finding the subset of heliostats to activate. It uses a genetic algorithm that works with binary strings in which value 0 at position $i$ means that heliostat $i$ will not be activated. Analogously, value 1 has the opposite meaning. Regarding its workflow, the procedure starts by generating a random initial population. The number of active heliostats is bounded after scanning the total power contained in the reference and the offer of heliostats according to the model. The minimum number of active heliostats for any individual results from accumulating the estimated power contribution of the most powerful ones until reaching that contained in the reference. The maximum number of active 
heliostats for any individual is computed by repeating the previous accumulation but with the least powerful heliostats according to the model and considering $25 \%$ of their estimated power contribution as if they aimed at a corner. The interested reader can find further details about the bounds in [17]. After that, it follows a classic evolutionary loop. Each loop iteration involves the following stages: i) parent selection, ii) reproduction, iii) spring mutation and iv) replacement. When the loop finishes after a given limit of iterations, the optimizer returns the best individual found as the solution.

Every progenitor is selected as the best individual out of a random sample, which is called tournament-based selection. The reproduction is through uniform crossover by generating a random binary reproduction mask and two descendants from every pair. The first descendant takes its binary values from the first progenitor at the positions in which the binary mask is 1 and from the second one elsewhere. The second one is built by inverting this rule. Mutation consists in randomly activating and deactivating heliostats on the offspring and re-evaluating. Finally, the population for the next iteration is formed by selecting the best individuals from the current one and the descendants, which is called elitism.

Since the genetic optimizer works with binary strings, it also needs a way to automatically generate full solutions that can be evaluated in terms of the objective function during the search. Thus, the optimizer also includes a dedicated module that completes any binary string by assigning a valid aim point to each active heliostat. It is called the auto-aiming module and serves to produce final flux maps that can be compared to the reference. This module works by iteratively aiming the most powerful active heliostat to the highest difference peak between the reference flux map and the predicted one.

The second layer works with the best solution found by the previous one. It tries to improve the aim point assignment heuristically made by the auto-aiming module. To do so, it applies a gradient descent optimizer that exploits the analytical formulation of the problem. The selection of active heliostats is not further changed at this point. This part focuses on improving the initial and heuristic aim point selection to minimize the objective function. The previous layer does not work in a continuous search space, but in a discrete one: the autoaiming module is limited to the discretization of the input reference. In contrast to it, the second layer is not limited in that way.

\section{Heuristic Control Strategy}

The method described in the previous section fulfils its requirements at the expense of a high computational cost, which is incompatible with on-line operation [15]. Moreover, it only works in a theoretical framework, and perfect 
modelling does not exist. Therefore, although the previous tools serve to configure the target field, different discrepancies may appear between the theoretical result and the flux map achieved when translating it to the plant. The control logic described in this section tries to overcome this problem.

The feedback control system developed in this work starts after applying the configuration computed for the plant by the model-based flux optimizer described in Section 3.2. It takes from the referred optimizer both the heliostats subset and their aim points as its initial state. Nevertheless, the field model is still taken into account: it serves to predict the effect of the changes proposed by the control logic between iterations before real feedback is available. Figure 2 summarizes the design approach. As shown, the system is intended for operating in closed loop: The configuration is applied, compared to the setpoint and iteratively corrected according to the feedback.

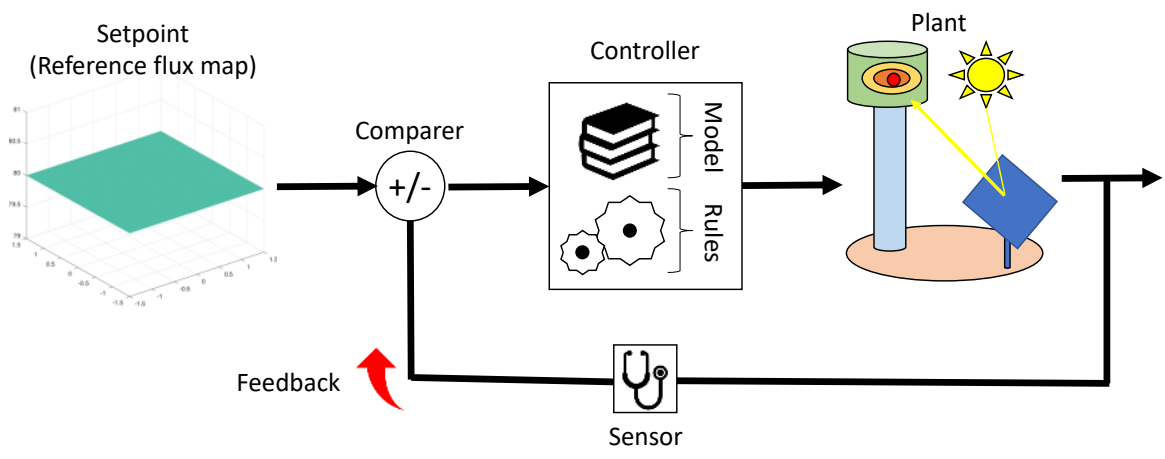

Figure 2

Design of a closed-loop controller

The control strategy considers the following three actions to improve the achieved flux distributions: i) Activating new heliostats, ii) Deactivating heliostats and iii) Changing the aim point of active heliostats.

The activation of heliostats is carried out when the difference between the total power contained in the setpoint and that reflected on the receiver is greater than a given threshold. In that situation, a new heliostat will be activated and aimed at the receiver. The process is repeated while the triggering condition is met and using the field model to update the flux map between iterations. After that, the system applies the changes and waits for new feedback to decide what to do again. The procedure is described in Algorithm 1. It takes as input the setpoint, the achieved flux map, the activation threshold ThrA (from Threshold of Activation), and a parameter Max_Scope, which is explained below. 
Algorithm 1

Steps for activating new heliostats by the controller

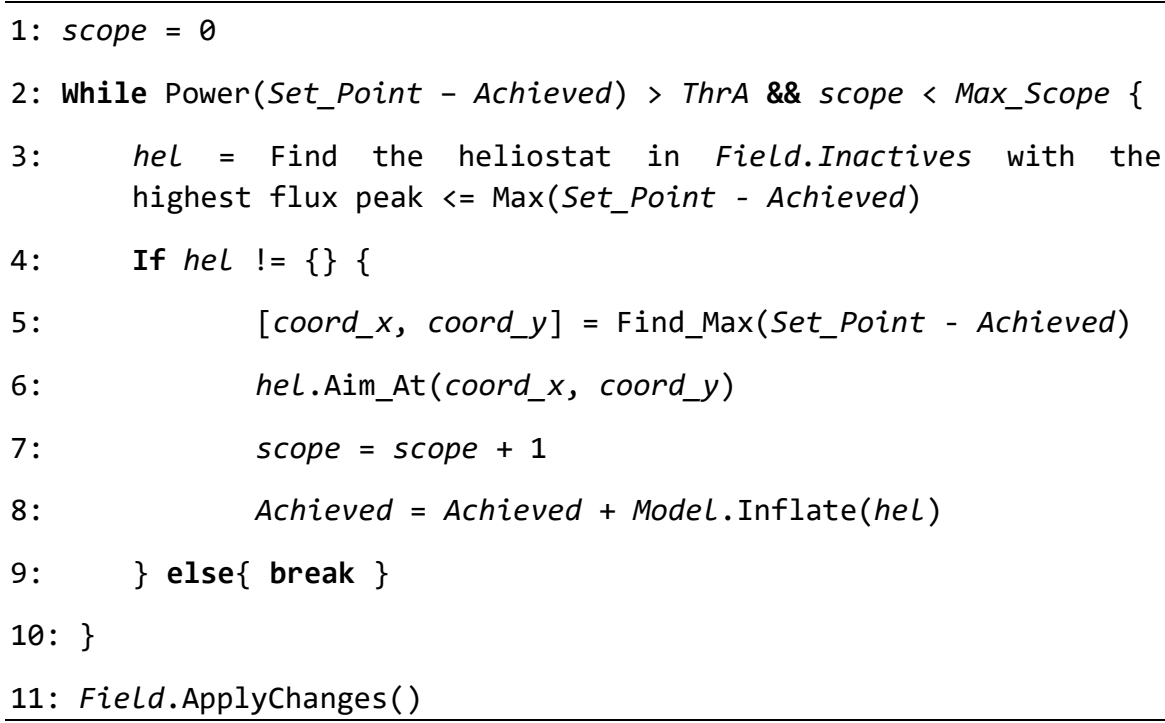

As shown, the heliostat selection criterion requires that its flux peak is as close as possible, without exceeding, to the greatest difference between the setpoint and the achieved flux map. Every new heliostat is aimed at the point with the highest difference in flux density, which tries to compensate for the potential lack of power. However, the activation logic might decide not to add any heliostat. This happens when no heliostat with an appropriate flux peak is found. Adding that type of heliostat could generate further discrepancies between the setpoint and the achieved maps. It is also necessary to highlight that the achieved or measured map is filtered to minimize noise. Otherwise, noisy singular points can misguide the control action. Furthermore, since the heliostat selection and the estimation of the effect of changes are based on the field model, no more than "Max_Scope", a limited and user-given number of heliostats, can be added. This approach avoids making too many changes without feedback, which would introduce back the limitations of modelling. The appropriate number of virtual or model-based changes allowed must be adjusted after a preliminary tuning stage and depends on the quality of the model. Finally, notice how the achieved flux map must be updated according to the model for the next iteration as done at line 8 .

Analogously, the system might consider that there are too many active heliostats, and it is necessary to deactivate some of them to reach the setpoint. It does not matter if they were part of the initial state or loaded in a previous activation stage. This action is triggered when there is more power on the receiver than in the setpoint, and the difference is greater than a given threshold as before. In that situation, the active heliostat with the nearest aim point to the greatest difference, 
and the lowest flux peak that does not exceed it, will be deactivated. The process is repeated while the triggering condition is met and limiting the maximum number of changes allowed again. After that, the system applies the changes and waits for new feedback to make a new decision. The procedure is detailed in Algorithm 2. It takes the same input as the previous one with the only exception of the threshold parameter, which is now labelled as ThrD (from Threshold for Deactivation) to differentiate it from the previous one. The achieved flux map is synthetically updated at line 7 in this case.

Algorithm 2

Steps for deactivating heliostats by the controller

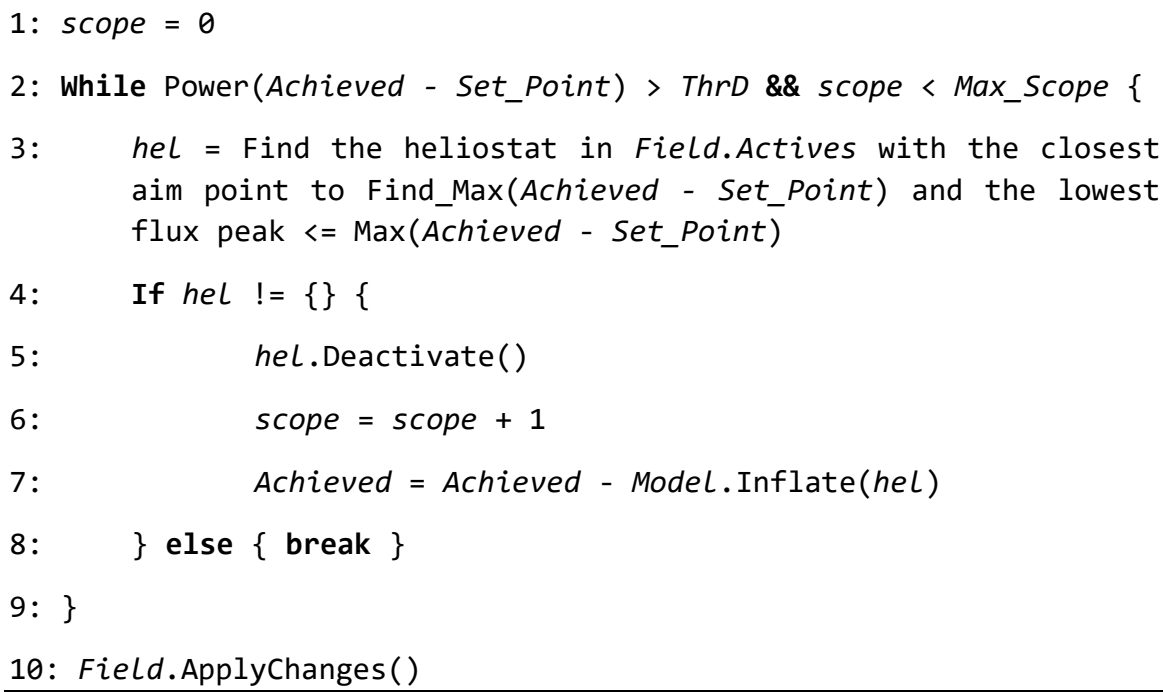

Finally, if no heliostat has been either activated or deactivated, the power balance is considered acceptable. Then, the controller studies the necessity of changing the aim points of the active heliostats. In this case, the focus is on the total power instead of on the differences in flux density.

The process starts by comparing the achieved flux map and the setpoint. The maximum and minimum differences are identified. The maximum difference indicates a region of the receiver in which there is more flux density than desired. On the contrary, the minimum difference means that there is less power there than expected. After identifying both points, the system seeks the heliostat that is aiming at the closest point to where the maximum difference is, and with a flux peak lower or equal to the minimum difference. If any, it aims that heliostat from its current point to where the minimum difference is. The process is repeated while the triggering condition is met and relying on the model-based updates to estimate the effect of changes. Finally, the system commits the changes to the 
plant and waits for new feedback to decide what to do again. Algorithm 3 contains a detailed description of the method.

Algorithm 3

Steps for re-aiming heliostats by the controller

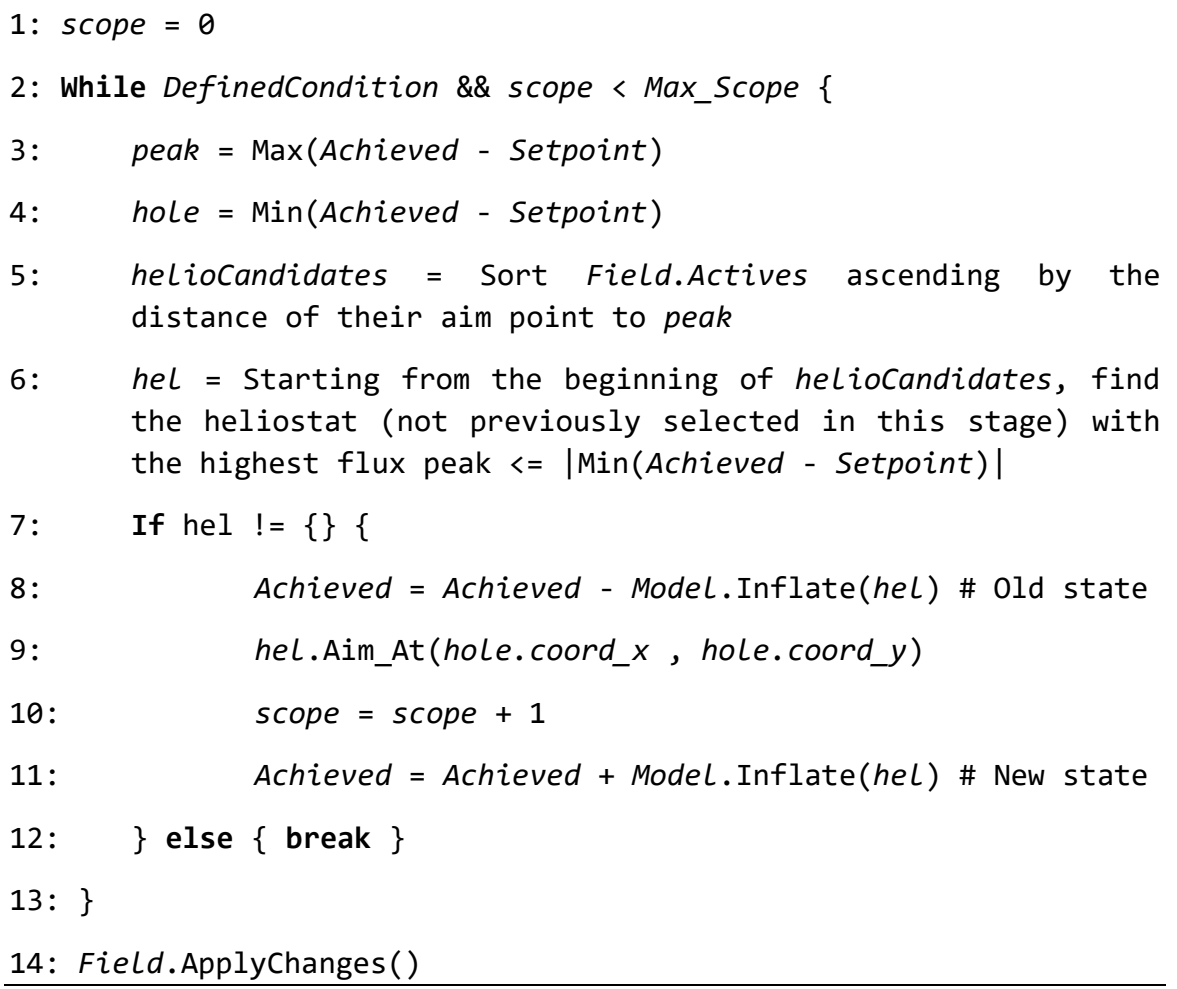

Some ideas from the previous algorithm must be further explained. Firstly, aside from not moving too many heliostats without feedback, which must be tuned as introduced, the triggering condition of this process also considers a configurable rule. This can be defined in general terms, e.g., the maximum root-mean-square error value between the setpoint and the achieved map, or with a more specific condition (such as the maximum standard deviation when looking for homogeneity). Secondly, as can be seen at line 6, re-aiming the same heliostat several times is not permitted to avoid unproductive changes and loops. Thirdly, as in the previous cases, the model serves to estimate the effect of changes. However, it is used twice this time. First, at line 8, the effect of not aiming the selected heliostat is estimated by subtracting its predicted flux map to the achieved one. After that, at line 11, the effect of re-aiming that heliostat is simulated by adding its flux map centered on its new aim point. Finally, the method might not 
change anything at a certain point. This does not mean that the achieved flux map is a perfect replica of the setpoint, but that the controller considers that it cannot improve the current result.

After having described the instantaneous control actions, there are also some dynamics concerns to consider when the method runs continuously. Chattering may appear, i.e., some heliostats are activated and deactivated several times from a time step to the other. This issue is addressed by defining some dwell time that restricts the minimum time allowed between two switches in the state of every heliostat.

\section{Experimentation and Results}

This section describes the experimentation carried out to assess the performance of the developed controller. The target field is the CESA-I, which belongs to Plataforma Solar de Almería (PSA) (Spanish for Solar Platform of Almería).

The field is in the south-east of Spain, at location $37^{\circ} 5^{\prime} 30^{\prime \prime} \mathrm{N}, 2^{\circ} 21^{\prime} 30^{\prime \prime} \mathrm{W}$. It has 300 heliostats which are north of the receiver. Each one of them has 12 facets $\left(3.05 \times 1.1 \mathrm{~m}^{2}\right.$ each) and a total surface close to $40 \mathrm{~m}^{2}$. The heliostats are deployed in a nearly flat surface with a slope of $0.9 \%$ rising from the tower towards the north. The receiver, which will be treated as a flat $3 \times 3 \mathrm{~m}^{2}$ square sampled at 0.04 $\mathrm{x} 0.04 \mathrm{~m}^{2}$ steps for defining the setpoint, is at $86.6 \mathrm{~m}$ above the ground. It is due north and tilted $33^{\circ}$ towards the field. The receiver is on a cylindrical tower $5 \mathrm{~m}$ in diameter and approximately $80 \mathrm{~m}$ in height. Figure 3 shows the field distribution from the tower base (left) and a real picture taken from the tower (right).

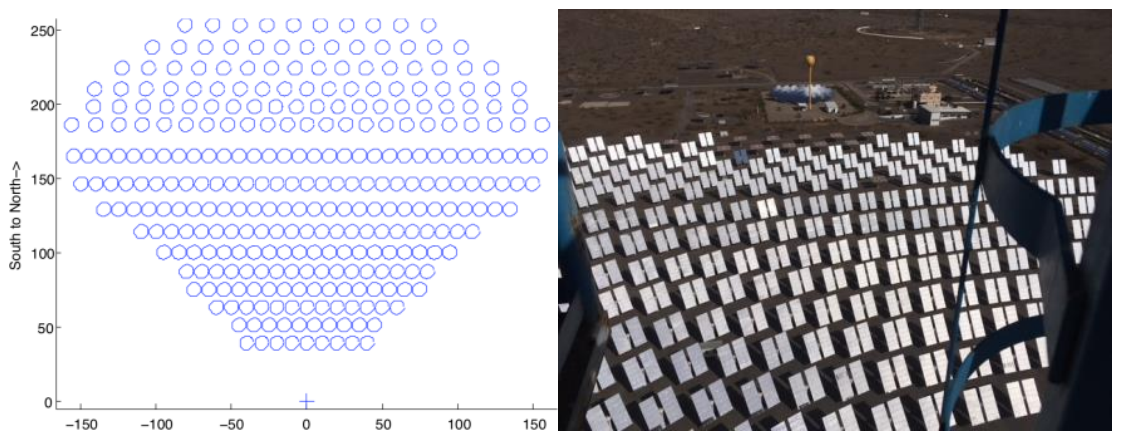

Figure 3

Design of the heliostat field CESA-I (left) and real picture of it from the tower (right)

The solar field has been modelled with the ray-tracing software Tonatiuh, which is considered as the reality, in this work, for practical reasons. Further information regarding this model can be found in [33]. 
The date considered is $21^{\text {st }}$ June, 2018, at 12:00 (local time). The measured DNI is $745.467 \mathrm{~W} / \mathrm{m}^{2}$. The goal is to produce a homogeneous flux distribution of 80 $\mathrm{kW} / \mathrm{m}^{2}$ and $739.6 \mathrm{~kW}$ over the receiver, which is a popular kind of setpoint in the literature. Figure 4 shows the setpoint defined for the model-based flux optimizer (left), the result that it achieves according to the model (right), and its real shape after applying the result to reality (bottom-middle). As can be seen, the theoretical result fulfils the requirements: The model-based flux optimizer automatically activates and aims 60 heliostats achieving a flux distribution in range [78, 82] $\mathrm{kW}$. It takes approximately 222 seconds, and the theoretical result contains $739.1 \mathrm{~kW}$ with a Standard Deviation (STD) of $1.2 \mathrm{~kW} / \mathrm{m}^{2}$ (that of the setpoint is $0 \mathrm{~kW} / \mathrm{m}^{2}$ ).

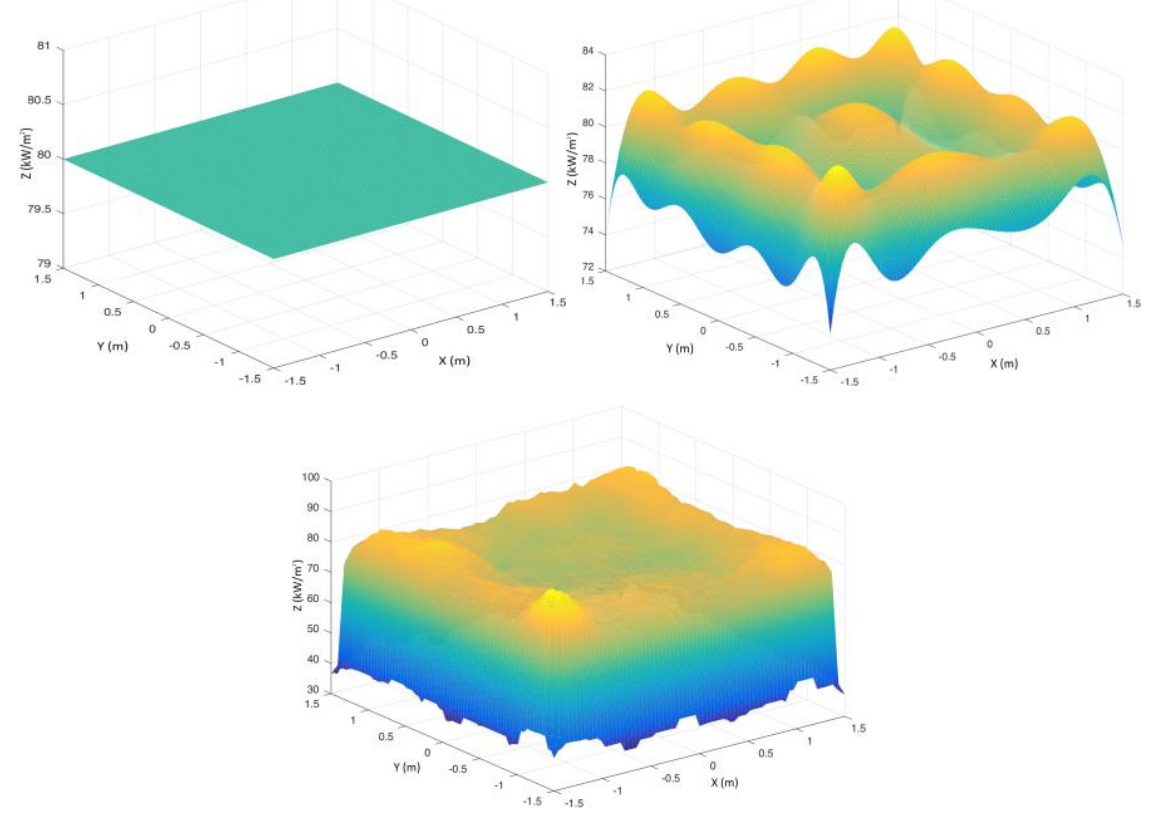

Figure 4

Setpoint to achieve on the receiver (left), theoretical result of the optimizer (right) and real output of the plant under that configuration (bottom-middle)

Logically, when the computed configuration is translated to reality (Figure 4, bottom-middle), the quality decreases. It contains $708.4 \mathrm{~kW}$ and its STD is 3.5 $\mathrm{kW} / \mathrm{m}^{2}$. The result is valid, especially considering the yearly scope of the field model and the general design of the model-based flux optimizer. It is even better, i.e., STD from 3.5 to $2.6 \mathrm{~kW} / \mathrm{m}^{2}$, if the metrics focus on the central zone to coincide with the real receiving area. Nevertheless, the result is expected to improve with the designed controller. For instance, the flux density in a corner is over the setpoint, while the central zone is below it. 
The controller has been tuned as follows: The maximum number of heliostats that can be activated, deactivated or moved without obtaining new feedback, i.e., Max_Scope, is set to 4 . The significant difference in power between the setpoint and the achieved flux map is set to $8 \mathrm{~kW}$ for ThrA and $2.4 \mathrm{~kW}$ for ThrD. These values are based on the total power contributions estimated by the field model at the considered instant and on the fact that deactivating might be more urgent, i.e., ThrD $<$ ThrA. Finally, the ad-hoc triggering condition to launch the re-aiming action depends on the STD of the achieved flux map. It is launched with values greater than 2.0 in the central zone.

The controller starts by adding two heliostats to the initial 60 . This first change increases the total power from 708.4 to $739.2 \mathrm{~kW}$, which is almost equal to the target power. The overall STD is improved from 3.5 to $3.4 \mathrm{~kW} / \mathrm{m}^{2}$ (2.6 to 2.1 $\mathrm{kW} / \mathrm{m}^{2}$ in the central area). Note that the power predicted with the model after updating was $740.4 \mathrm{~kW}$, and the overall and central STD values were 3.3 and 2.2 $\mathrm{kW} / \mathrm{m}^{2}$, respectively. Thus, the model was useful to estimate the effect of the changes applied.

At the second step, the controller opts for changing the aim points of some of the active heliostats. Specifically, it executes four iterations of its internal loop that moves heliostats from the hottest zones to those with less power. However, it is only able to move three heliostats in the end. The last iteration fails to find an active heliostat with flux peak as low as required and different from those chosen in the three previous iterations. After applying these changes, there are $742.2 \mathrm{~kW}$ on the receiver, i.e., only $0.35 \%$ higher than the $739.6 \mathrm{~kW}$ of the setpoint. The overall STD is improved from 3.4 to $3.0 \mathrm{~kW} / \mathrm{m}^{2}$, and from 2.1 to $1.7 \mathrm{~kW} / \mathrm{m}^{2}$ in the central zone. This time, the behavior predicted by the model after applying the changes, i.e., moving three heliostats, where $742.0 \mathrm{~kW}$ in power, and overall and central STD values of 3.0 and $1.6 \mathrm{~kW} / \mathrm{m}^{2}$, respectively. Therefore, the field model is not only useful for initial the model-based flux optimizer, but also for the controller to synchronize the changes proposed over gathered the flux map.

Figure 5 shows the flux distribution obtained after applying the changes of the controller over the initial field configuration up to the second step. As can be seen, the flux distribution is homogeneous in general. The effect of reducing the STD values from 3.5 and 2.6 to 3.0 and $1.7 \mathrm{~kW} / \mathrm{m}^{2}$, respectively, is evident. It is clearly better than the initial flux map before applying the control changes (Figure 4, bottom-middle), especially considering the depression of the central zone in that flux map. In fact, the total power is now almost identical to the target, i.e., 739.6 $\mathrm{kW}$, namely, $742.2 \mathrm{~kW}$ after the controller versus the initial value of $708.4 \mathrm{~kW}$.

Finally, the deactivation stage is triggered with the previous input. It deactivates one heliostat to decrease the highest flux peak at the bottom left corner. After doing so, the method leaves 61 active ones. The result achieved is shown in Figure 6 and, as can be seen, it is even better than the previous one: the bottom left flux peak is significantly less pronounced. In fact, the visualization axes were 
automatically reduced from 100 to $90 \mathrm{~kW} / \mathrm{m}^{2}$. At this point, the total power is slightly lower than desired, i.e., $736.4 \mathrm{~kW}$, but still almost identical to the target of $739.6 \mathrm{~kW}$. Moreover, the STD values are now $2.9 \mathrm{~kW} / \mathrm{m}^{2}$ for the overall shape and $1.5 \mathrm{~kW} / \mathrm{m}^{2}$ for the central area. In this case, the model predicted $735.0 \mathrm{~kW}$ in power and 3.0 and $1.6 \mathrm{~kW} / \mathrm{m}^{2}$ in overall and central STD, which are near to the values achieved in the reality.

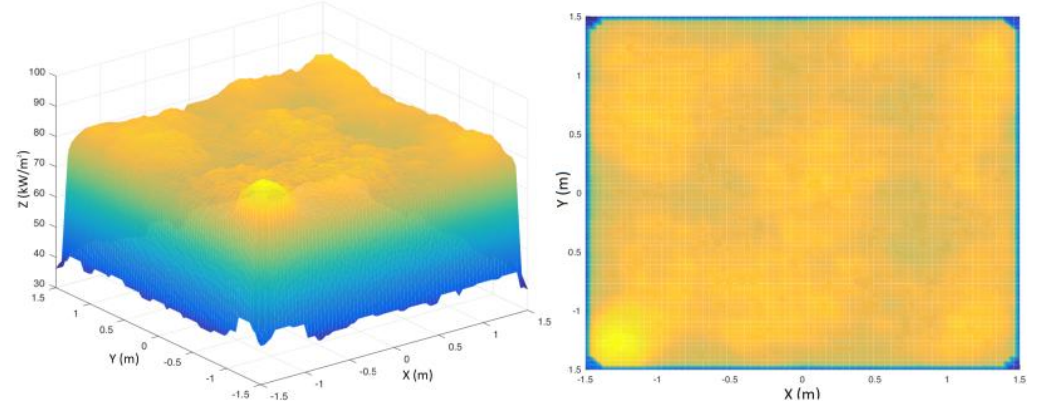

Figure 5

Flux distribution achieved on the receiver surface after applying the two changing steps proposed by the designed controller. Overall (left) and top (right) views

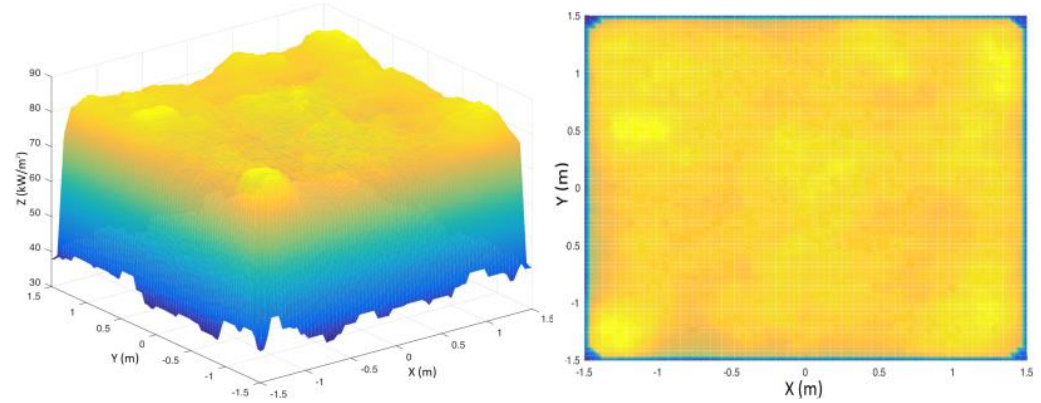

Figure 6

Flux distribution achieved on the receiver surface after applying the three changing steps proposed by the designed controller. Overall (left) and top (right) views

After the previous change, the measured feedback does not trigger any additional action of the proposed controller. The difference in power is not big enough to consider activating or deactivating, and the STD in the center is also in the desired range. Thus, the controller has only executed 3 phases, and it has improved the initial and model-based starting point. The total power has raised from 708.4 to $736.4 \mathrm{~kW}(739.6 \mathrm{~kW}$ was desired), and the overall STD has been reduced from 3.5 to 2.9 and from 2.6 to $1.5 \mathrm{~kW} / \mathrm{m}^{2}$ in the overall shape and in the center, respectively. 


\section{Conclusions}

Controlling the flux distribution that a heliostat field produces on its receiver is important. It affects the efficiency and safety of the plant, and even its production costs, considering the expenses associated with the receiver. Unfortunately, it is also a challenging problem that involves selecting the heliostats to use and their aim points. There are different methods to address this problem in the literature. While most of them focus on adjusting the aim point and obtaining homogeneous flux distributions, the authors of this work have proposed a general flux optimization method linked to a new modelling strategy. Its theoretical results fulfil the requirements, but their quality worsens when translated to the plant due to inherent modelling errors.

This paper proposes a simple feedback controller that can perform three different actions: activating, deactivating and re-aiming heliostats. The decision between the two first options depends on the difference in power between the setpoint and the obtained flux map. The activation of the third one is adapted to the goal and based on comparing flux densities between the setpoint and the achieved flux map. The process combines real feedback with an analytical field model. The control logic has been added to the workflow defined by the flux map optimizer and its associated field model. It compensates for internal modelling errors by making a few changes based on the input flux map.

In the experimentation carried out, the setpoint has been set to a homogeneous flux distribution, which is the most studied target in the literature. According to the results obtained, the control logic improves the flux distribution that results from directly applying the configuration computed by the model-based flux optimizer. Considering that the setpoint has $739.6 \mathrm{~kW}$ and $0 \mathrm{~kW} / \mathrm{m}^{2}$ in standard deviation, the controller raises the achieved power from 708.4 to $736.4 \mathrm{~kW}$ and reduces the central standard deviation from 2.6 to $1.5 \mathrm{~kW} / \mathrm{m}^{2}$.

As future work, the control strategy will be tested considering the apparent solar movement instead of a fixed point in time. The software package Tonatiuh will also be replaced with real measurements when they can be taken from the target field.

\section{Acknowledgement}

This work has been supported by the Spanish Ministry of Economy, Industry and Competitiveness under grants RTI2018-095993-B-100 and DPI2017-85007-R, and the Spanish Junta de Andalucía under grant UAL18-TIC-A020-B, co-funded by FEDER funds. N. C. Cruz has been supported by an FPU fellowship from the Spanish Ministry of Education under grant FPU14/01728.

\section{References}

[1] S. Kiwan and S. Al Hamad, "On analyzing the optical performance of solar central tower systems on hillsides using biomimetic spiral distribution", Journal of Solar Energy Engineering, Vol. 141, No. 1, pp. 1-12, 2019 
[2] J. Wang, L. Duan and Y. Yang, "An improvement crossover operation method in genetic algorithm and spatial optimization of heliostat field," Energy, Vol. 155, pp. 15-28, 2018

[3] K. Wang, Y. L. He, Y. Qiu and Y. Zhang, "A novel integrated simulation approach couples MCRT and Gebhart methods to simulate solar radiation transfer in a solar power tower system with a cavity receiver," Renewable Energy, Vol. 89, pp. 93-107, 2016

[4] M. Saghafifar, M. Gadalla, and K. Mohammadi, "Thermo-economic analysis and optimization of heliostat fields using AINEH code: Analysis of implementation of non-equal heliostats (AINEH)," Renewable Energy, Vol. 135, pp. 920-935, 2019

[5] V. S. Reddy, S. C. Kaushik, K. R. Ranjan and S. K. Tyagi, "State-of-the-art of solar thermal power plants - A review," Renewable and Sustainable Energy Reviews, Vol. 27, pp. 258-273, 2013

[6] F. J. Collado and J. Guallar, "Fast and reliable flux map on cylindrical receivers," Solar Energy, Vol. 169, pp. 556-564, 2018

[7] C. J. Noone, A. Ghobeity, A. H. Slocum, G. Tzamtzis and A. Mitsos, "Site selection for hillside central receiver solar thermal plants," Solar Energy, Vol. 85, No. 5, pp. 839-848, 2011

[8] S. Alexopoulos and B. Hoffschmidt, "Advances in solar tower technology," WIREs Energy and Environment, Vol. 6, No. 1, pp. 1-19, 2017

[9] D. Y. Goswami, Principles of Solar Engineering ( $3^{\text {rd }}$ Ed). Taylor \& Francis, 2015

[10] A. Salomé, F. Chhel, G. Flamant, A. Ferrière and F. Thiery, "Control of the flux distribution on a solar tower receiver using an optimized aiming point strategy: Application to THEMIS solar tower," Solar Energy, Vol. 94, pp. 352-366, 2013

[11] M. Astolfi, M. Binotti, S. Mazzola, L. Zanellato and G. Manzolini, "Heliostat aiming point optimization for external tower receiver," Solar Energy, Vol. 157, pp. 1114-1129, 2017

[12] K. Wang, Y. L. He, X. D. Xue and B. C. Du, "Multi-objective optimization of the aiming strategy for the solar power tower with a cavity receiver by using the non-dominated sorting genetic algorithm," Applied Energy, Vol. 205, pp. 399-416, 2017

[13] S. M. Besarati, D. Y. Goswami and E. K. Stefanakos, "Optimal heliostat aiming strategy for uniform distribution of heat flux on the receiver of a solar power tower plant," Energy Conversion and Management, Vol. 84, pp. 234-243, 2014

[14] A. Grobler, "Aiming strategies for small central receiver systems," Master's degree dissertation, Stellenbosch University, 2015 
[15] T. Ashley, E. Carrizosa and E. Fernández-Cara, "Optimisation of aiming strategies in Solar Power Tower plants," Energy, Vol. 137, pp. 285-291, 2017

[16] A. Sánchez-González, M. R. Rodríguez-Sánchez and D. Santana, "Aiming factor to flatten the flux distribution on cylindrical receivers," Energy, Vol. 153, pp. 113-125, 2018

[17] N. C. Cruz, J. D. Álvarez, J. L. Redondo, M. Berenguel and P. M. Ortigosa, "A two-layered solution for automatic heliostat aiming," Engineering Applications of Artificial Intelligence, Vol. 72, pp. 253-266, 2018

[18] E. F. Camacho and A. J. Gallego, "Advanced control strategies to maximize ROI and the value of the concentrating solar thermal (CST) plant to the grid," in Advances in Concentrating Solar Thermal Research and Technology, Woodhead Publishing, Elsevier, 2017, Ch. 14, pp. 311-336

[19] L. Roca, R. Díaz-Franco, A. de la Calle, J. Bonilla, L. J. Yebra and A. Vidal, "A combinatorial optimization problem to control a solar reactor," Energy Procedia, Vol. 49, pp. 2037-2046, 2014

[20] A. Sánchez-González, M. R. Rodríguez-Sánchez, and D. Santana, "Aiming strategy model based on allowable flux densities for molten salt central receivers," Solar Energy, Vol. 157, pp. 1130-1144, 2017

[21] A. Grobler and P. Gauché, "A review of aiming strategies for central receivers," in Proceedings of the second Southern African Solar Energy Conference, pp. 1-8, 2014

[22] F. Glover and M. Laguna, Tabu Search. Kluwer, Norwell, MA, 1997

[23] D. E. Goldberg and J. H. Holland, "Genetic algorithms and machine learning," Machine Learning, Vol. 3, No. 2, pp. 95-99, 1998

[24] Q. Yu, Z. Wang and E. Xu, "Analysis and improvement of solar flux distribution inside a cavity receiver based on multi-focal points of heliostat field," Applied Energy, Vol. 136, pp. 417-430, 2014

[25] B. Belhomme, R. Pitz-Paal and P. Schwarzbözl, "Optimization of heliostat aim point selection for central receiver systems based on the ant colony optimization metaheuristic," Journal of Solar Energy Engineering, Vol. 136, No. 1, pp. 011005-011012, 2014

[26] M. Dorigo and C. Blum, "Ant colony optimization theory: A survey," Theoretical Computer Science, Vol. 344, No, 2. pp. 243-278, 2005

[27] D. Maldonado, R. Flesch, A. Reinholz and P. Schwarzbözl, "Evaluation of aim point optimization methods. In AIP Conference Proceedings," in AIP Conference Proceedings, Vol. 2033, No. 1, pp. 1-8, 2018 
[28] T. Ashley, E. Carrizosa and E. Fernández-Cara, "Inclement weather effects on optimal aiming strategies in solar power tower plants" in Proceedings of SolarPACES, DOI: 10.1063/1.5067041, 2018

[29] A. Kribus, "Closed loop control of heliostats," Energy, Vol. 29, No. 5-6, pp. 905-913, 2004

[30] M. R. Convery, "Closed-loop control for power tower heliostats," in Proceedings of SPIE, Vol. 8108, pp. 81080M-1, 2011

[31] J. Freeman and L. R. Chandran, " Closed loop control system for a heliostat field," in 2015 IEEE International Conference on Technological Advancements in Power and Energy, pp. 272-277, 2015

[32] A. J. Gallego and E. F. Camacho, "On the optimization of flux distribution with flat receivers: A distributed approach," Solar Energy, Vol. 160, pp. $117-129,2018$

[33] N. C. Cruz, R. Ferri-García, J. D. Álvarez, J. L. Redondo, J. FernándezReche, M. Berenguel, R. Monterreal and P. M. Ortigosa, "On building-up a yearly characterization of a heliostat field: A new methodology and an application example," Solar Energy, Vol. 173, pp. 578-589, 2018

[34] M. Blanco, A. Mutuberria, A. Monreal and R. Albert, "Results of the empirical validation of Tonatiuh at Mini-Pegase CNRS-PROMES facility," in Proceedings of SolarPACES, 2011

[35] C. H. Chen, C. C. Chung, F. Chao, C. M. Lin and I. J. Rudas, "Intelligent robust control for uncertain nonlinear multivariable systems using recurrent cerebellar model neural networks," Acta Polytechnica Hungarica, Vol. 12, No. 5, pp. 7-33, 2015

[36] L. Breiman, "Random forest," Machine Learning, Vol. 45, No. 1, pp. 5-32, 2001 